January 21, 2003

\title{
A NON-COMMUTATIVE POSITIVSTELLENSATZ ON ISOMETRIES
}

\author{
JOHN W. HELTON, SCOTT MC CULLOUGH, MIHAI PUTINAR
}

\begin{abstract}
A symmetric non-commutative polynomial $p$ when evaluated at a tuple of operators on a finite dimensional, real Hilbert space $H$ has a value which is a symmetric operator. We show that any such polynomial which takes positive semidefinite values on the variety $Z$ of spherical isometries is represented as a sum of squares of polynomials plus a residual part vanishing on $Z$. Here by spherical isometries we mean tuples $A=\left(A_{1}, A_{2}, \ldots, A_{n}\right)$ of operators on $H$ such that

$$
A_{1}^{T} A_{1}+\ldots+A_{n}^{T} A_{n}=I .
$$
\end{abstract}

This observation improves prior theorems known only for strictly positive polynomials. It is known that for commutative polynomials the result is false.

\section{INTRODUCTION}

The subject of positive polynomials on real algebraic varieties is related to Hilbert's 17-th problem and has a rather long and interesting history, see [2], [9]. A variety of decomposition theorems for regular positive functions on real algebraic or analytic varieties, known generically as Positivstellensatze, is known, for instance [14], [12], [1]. The distinction between decompositions of strictly positive functions and non-negative ones is sensible; only in recent years has a better understanding of the role of the geometry of the underlying (affine) variety in such theorems been revealed, see [12], [7], [13].

The natural correspondence between decompositions of polynomials into sums of squares and moment problems was known for a long time, see [6], [11]. The landmark contribution of Gilles Cassier [3] has established a new and powerful technique of deriving decompositions into sums of squares from simple separation arguments of convex sets. See for instance [10] and [13] for such applications.

Motivated by optimization problems, the first author has considered ([4]) sums of squares of polynomials in non-commutative variables. In this case the evaluations at tuples of matrices or operators are the analogue of point evaluations in the commutative situation. The subsequent paper [5] studies weighted decompositions into sums of squares of non-commuting polynomials which are non-negative on a "variety" of matrices. Not surprisingly, the statements and the proofs in the non-commutative case are simpler than the classical commutative ones. Indeed, the results in this paper are obtained by adaptations of Cassier's technique and are false in the commutative setting. 
The present note is a continuation of the work in [5]. Here we prove that a non-negative, non-commutative polynomial on a set of isometric tuples of operators can be decomposed into a sum of squares plus a necessary residual part. We indicate several alternate versions of our main result with spherical isometries replaced by other collections of operators. The fact that key sets are closed is proved at a general enough level to accomodate possible further applications.

\section{PRELIMINARIES}

This section contains a few general facts about Hilbert space representations of a finitely generated free algebra.

Let $n \geq 1$ be fixed and let $\mathcal{P}$ be the algebra of real coefficient polynomials in the non-commuting indeterminates $\{x, y\}=\left\{x_{1}, \ldots, x_{n}, y_{1}, \ldots, y_{n}\right\}$, where the $x_{j}$ and $y_{j}$ are adjoint pairs as we now explain. We consider the linear involution $a \mapsto a^{T}$ defined on the variables $x, y$ by $x_{j}^{T}=y_{j}$ and $y_{j}^{T}=x_{j}$; defined on words in the variables by

$$
\left(z_{i_{1}} z_{i_{2}} \ldots z_{i_{k}}\right)^{T}=z_{i_{k}}^{T} z_{i_{k-1}}^{T} \ldots z_{i_{1}}^{T},
$$

where $z \in\{x, y\}$; and extended to polynomials in $\mathcal{P}$ by linearity. The polynomial $p(x)$ is symmetric if $p(x)^{T}=p(x)$, that is, the coeffcients of a monomial and its adjoint are equal. Often in the sequel $y_{j}$ is identified with $x_{j}^{T}$.

An $n$-tuple $A=\left(A_{1}, A_{2}, \ldots, A_{n}\right)$ of bounded operators acting on the separable real Hilbert space $H$ determines the evaluation map

$$
\mathcal{P} \longrightarrow \mathcal{B}(H), \quad p \mapsto p(A),
$$

which is a unital ${ }^{T}$-algebra homomorphism.

For a subset $Z$ of tuples $A$ as above, define the associated ideal $I(Z)$ by

$$
I(Z)=\{p \in \mathcal{P} ; p(A)=0, \quad A \in Z\},
$$

let $\mathcal{P}(Z)$ denote the quotient $\mathcal{P} / I(Z)$ and let $\pi: \mathcal{P} \rightarrow \mathcal{P}(Z)$ denote the quotient mapping. Note that the quotient is an algebra which, as $I(Z)$ is invariant under the involution, inherits an involution from $\mathcal{P}$. Indeed, $\pi(p)^{T}=\pi\left(p^{T}\right)$. We regard the algebra $\mathcal{P}(Z)$ as a space of functions defined on $Z$.

Fix a degree $d \geq 0$ and denote by $\mathcal{P}_{d}$ the subset of all polynomials in $\mathcal{P}$ of degree less than or equal to $d$. Let

$$
\mathcal{P}_{d}(Z)=\pi\left(\mathcal{P}_{d}\right)=\frac{\mathcal{P}_{d}+I(Z)}{I(Z)}=\frac{\mathcal{P}_{d}}{\mathcal{P}_{d} \cap I(Z)} .
$$

Note, for $d^{\prime} \geq d$, the canonical inclusion

$$
\mathcal{P}_{d}(Z) \subset \mathcal{P}_{d^{\prime}}(Z)
$$

holds, so that if $\mathcal{P}_{d^{\prime}}(Z)$ is normed, then $\mathcal{P}_{d}(Z)$ is a closed subspace.

Define by $\mathcal{S} y m$ the subalgebra of $\mathcal{P}$ consisting of symmetric polynomials, and analogously $\mathcal{S}_{y m_{d}}, \mathcal{S}_{y m_{d}}(Z)$. 
Let

$$
\mathcal{C}_{2 d}=\operatorname{co}\left\{p^{T} p ; p \in \mathcal{P}_{d}\right\},
$$

where co denotes convex hull, and let

$$
\mathcal{C}_{2 d}(Z)=\pi\left(\mathcal{C}_{2 d}\right)=\frac{\mathcal{C}_{2 d}+I(Z)}{I(Z)} .
$$

Obviously, for an element $g \in \mathcal{C}_{2 d}(Z)$ and an $A \in Z$, the operator $g(A)$ is positive semi-definite (PSD); i.e., $g(A)$ is symmetric and $\langle g(A) h, h\rangle \geq 0$ for each $h \in H$ where $H$ is the Hilbert space on which each $A_{j}$ acts. The main result of the note consists of the converse to this observation under suitable additional hypothesis on $Z$.

\section{3. $\mathcal{C}_{2 d}(Z)$ IS CLOSED}

We shall use a Hahn-Banach argument to seperate a polynomial from the convex set $\mathcal{C}_{2 d}(Z)$. Thus it is important that $\mathcal{C}_{2 d}(Z)$ is closed and in this section we show that at a great level of generality. First we construct a finite dimensional faithful representation of $\mathcal{S} y m_{d}$.

Lemma 3.1. Assume that the set $Z$ of $n$-tuples of operators is invariant under joint unitary invariance. For every $d \geq 0$ there exists a basis $\beta_{1}, \beta_{2}, \ldots, \beta_{k}$ of the vector space $\mathcal{S} y m_{d}(Z)$ and a set $B_{1}, B_{2}, \ldots, B_{k}$ of tuples of operators belonging to $Z$ and acting on a finite dimensional Hilbert space $H$, so that, for a distinguished vector $\xi \in H$, the relations

$$
\left\langle\beta_{i}\left(B_{j}\right) \xi, \xi\right\rangle=\delta_{i j},
$$

hold, where $\delta_{i j}$ is Kronecker's symbol.

Proof. We will use several times the following elementary observation: if $A$ is a bounded symmetric operator acting on a real Hilbert space $H$, then $A \neq 0$ if and only if there exists a vector $v \in H$ such that $\langle A v, v\rangle \neq 0$.

Let $g_{1}, g_{2}, \ldots, g_{k}$ be a basis of $\mathcal{S} y m_{d}(Z)$. Subsequently we will perform certain linear operations on the vectors $g_{j}$ but, for simplicity, still denote them with $g_{j}$. Since $g_{1} \neq g_{2}$ there exists an element $B_{1} \in Z$ such that $g_{1}\left(B_{1}\right) \neq g_{2}\left(B_{1}\right)$. Hence there exists a vector $\xi \in H$ with the properties:

$$
\left\langle g_{1}\left(B_{1}\right) \xi, \xi\right\rangle \neq\left\langle g_{2}\left(B_{1}\right) \xi, \xi\right\rangle, \quad 0 \neq\left\langle g_{1}\left(B_{1}\right) \xi, \xi\right\rangle .
$$

By replacing $g_{1}, g_{2}$ by a linear combination of these vectors we can assume that:

$$
\left\langle g_{1}\left(B_{1}\right) \xi, \xi\right\rangle=1, \quad\left\langle g_{2}\left(B_{1}\right) \xi, \xi\right\rangle=0 .
$$

Because the element $g_{2}$ is non-zero, there exists $K_{2}$ in $Z$ and a vector $\eta_{2} \in H$ such that $\left\langle g_{2}\left(K_{2}\right) \eta_{2}, \eta_{2}\right\rangle=1$. Choose now a unitary transformation $U$ on $H$ such that $U \eta_{2}=\xi$, and denote $B_{2}=U K_{2} U^{T}$, where the unitary conjugation on the tuple $K_{2}$ is applied entry by entry. Whence, $\left\langle g_{2}\left(B_{2}\right) \xi, \xi\right\rangle=1$. Replace $g_{2}$ by a linear combination of the form $g_{2}+\alpha g_{1}$ to obtain:

$$
\left\langle g_{2}\left(B_{1}\right) \xi, \xi\right\rangle=0, \quad\left\langle g_{2}\left(B_{2}\right) \xi, \xi\right\rangle=1 .
$$


In a similar manner, starting from $\left\langle g_{3}\left(K_{3}\right) \eta_{3}, \eta_{3}\right\rangle=1$ one can find a tuple $K_{3} \in Z$ such that $\left\langle g_{3}\left(K_{3}\right) \xi, \xi\right\rangle=1$. Replace it by a linear combination of the form $g_{3}+\alpha g_{2}+\gamma g_{3}$ to obtain:

$$
\left\langle g_{3}\left(B_{i}\right) \xi, \xi\right\rangle=0, \quad\left\langle g_{3}\left(K_{3}\right) \xi, \xi\right\rangle=1, \quad i=1,2 .
$$

Putting all these relations together we have constructed another basis $g_{1}, g_{2}, \ldots, g_{k}$ of $\mathcal{P}_{2 d}(Z)$ and elements $B_{1}, B_{2}, \ldots, B_{k} \in Z$, together with a vector $\xi \in H$, such that:

$$
\left\langle g_{j}\left(B_{i}\right) \xi, \xi\right\rangle=0, \quad\left\langle g_{j}\left(B_{j}\right) \xi, \xi\right\rangle=1, \quad i<j \leq k .
$$

Finally, by choosing $\beta_{j}$ equal to a linear combination of $g_{j}, g_{j+1}, \ldots, g_{k}$ we can fulfill (1) by inductive elimination.

As a consequence of Lemma 3.1,

$$
|\pi(h)|=\sum_{j=1}^{k}\left|\left\langle h\left(B_{j}\right) \xi, \xi\right\rangle\right|, \quad h \in \mathcal{S} y m_{d},
$$

defines a norm on the finite vector space $\mathcal{S}_{y} m_{d}(Z)$. Moreover, if $d$ is even and $h \in \mathcal{C}_{d} \subset \mathcal{S} y m_{d}$, then

$$
|\pi(h)|=\sum_{j=1}^{k}\left\langle h\left(B_{j}\right) \xi, \xi\right\rangle,
$$

because $\left\langle f(A)^{T} f(A) \xi, \xi\right\rangle=\|f(A) \xi\|^{2} \geq 0$.

As for the spaces $\mathcal{P}_{c}(Z), c \leq d / 2$, we can define the norm:

$$
\|f\|^{2}=\left|f^{T} f\right|, \quad f \in \mathcal{P}_{c}(Z), c \leq d / 2 .
$$

For future reference, note that, for $f, g \in \mathcal{P}_{c}(Z)$ standard calculations show

$$
\left|f^{T} f-g^{T} g\right| \leq\|(f-g)\|(\|f\|+\|g\|) .
$$

It is important to note that the above constructions, in particular the finite dimensional real Hilbert space $H$, where the operators $B_{j}$ act, depend on $d$.

A set (or class) $Z$ of tuples of operators is closed under joint unitary transformation if whenever $A=\left(A_{1}, \ldots, A_{n}\right)$ is in $Z$ is a tuple acting on the Hilbert space $H, K$ is a Hilbert space, and $U: H \rightarrow K$ is unitary, then

$$
U^{T} A U=\left(U^{T} A_{1} U, U^{T} A_{2} U, \ldots, U^{T} A_{n} U\right)
$$

is in $Z$.

Lemma 3.2. Assume that the set $Z$ of tuples of operators is invariant under joint unitary transformations. Then for every $d \geq 0$ the cone $\mathcal{C}_{2 d}(Z)$ is closed in $\mathcal{P}_{2 d}(Z)$.

Proof. This is an application of Carathéodory's theorem, see for instance [11] for a similar derivation in the commutative case. Namely, if $k$ denotes the dimension of $\mathcal{S} y m_{2 d}$, then Carathéodory's theorem says every element $h$ 
in the convex cone $\mathcal{C}_{2 d}$ can be written as a combination of at most $k$ elements from the set $\left\{f^{T} f: f \in \mathcal{P}_{d}\right\}$. Thus, there exists $f_{1}, \ldots, f_{k} \in \mathcal{P}_{d}$ such that

$$
h=f_{1}^{T} f_{1}+f_{2}^{T} f_{2}+\ldots+f_{k}^{T} f_{k} .
$$

Suppose $h^{\nu} \in \mathcal{C}_{2 d}, \nu \in \mathbf{N}$, is such that the sequence $\pi\left(h^{\nu}\right)$ is Cauchy in

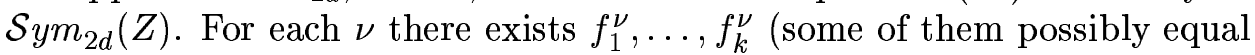
to zero) such that

$$
h^{\nu}=\sum_{j=1}^{k} f_{j}^{\nu T} f_{j}^{\nu}
$$

Using the norm $|*|$,

$$
\left|\pi\left(h^{\nu}\right)\right|=\sum_{j=1}^{k} \sum_{\ell=1}^{k}\left\langle\left(f_{j}^{\nu}\left(B_{\ell}\right)\right)^{T} f_{j}^{\nu}\left(B_{\ell}\right) \xi, \xi\right\rangle=\sum_{j=1}^{k}\left\|\pi\left(f_{j}^{\nu}\right)\right\|^{2} .
$$

Since $\pi\left(h^{\nu}\right)$ is Cauchy, it follows from (3) that for each $j$ the sequence $\left\{\pi\left(f_{j}^{\nu}\right)\right\}_{\nu}$ is bounded in the norm (2) of the finite dimensional space $\mathcal{P}_{d}(Z)$. Therefore, by passing to a common subsequence we can assume that for each $j,\left\{\pi\left(f_{j}^{\nu}\right)\right\}_{\nu}$ is a convergent sequence in $\mathcal{P}_{d}(Z)$. Let $g_{j}=\lim _{\nu} \pi\left(f_{j}^{\nu}\right) \in \mathcal{P}_{d}(Z)$ and choose $f_{j} \in \mathcal{P}_{d}$ such that $\pi\left(f_{j}\right)=g_{j}$.

From (3) it follows that there exists a universal constant $\gamma>0$ with the property that, for $j=1,2, \ldots, k$,

$$
\left|\pi\left(f_{j}^{\nu T} f_{j}^{\nu}\right)-\pi\left(f_{j}^{T} f_{j}\right)\right| \leq \gamma\left\|\pi\left(f_{j}^{\nu}-f_{j}\right)\right\| .
$$

Consequently, the limit $f$ of the sequence $\pi\left(h^{\nu}\right)$ in the norm $|\cdot|$ is of the form $\pi(f)$, where

$$
f=f_{1}^{T} f_{1}+\ldots+f_{k}^{T} f_{k}
$$

and this proves that the cone $\mathcal{C}_{2 d}(Z)$ is closed.

\section{MAin RESUlt}

4.1. Spherical isometries. The notation of the previous section will be used throughout the rest of the note. Basically, $\mathcal{P}$ is the space of polynomials in the non-commuting variables $\left(x, x^{T}\right)=\left\{x_{1}, x_{2}, \ldots, x_{n}, x_{1}^{T}, \ldots, x_{n}^{T}\right\}$, while $Z$ is a set of $n$-tuples of bounded operators acting on an arbitrary finite dimensional Hilbert space $H$, and $I(Z)$ is the ideal of polynomials vanishing on $Z$.

A tuple $A=\left(A_{1}, \ldots, A_{n}\right)$ of operators on a finite dimensional, real Hilbert space $H$ is an isometric tuple or spherical isometry provided $\sum A_{j}^{T} A_{j}=I$. The collection of all isometric tuples is not actually a set, due to the ambiguity in choosing the Hilbert space $H$, but this causes no logical difficulties.

Theorem 4.1. Let $Z$ be the class of spherical isometries $\left(A_{1}, A_{2}, \ldots, A_{n}\right)$, i.e. $A_{1}^{T} A_{1}+\ldots+A_{n}^{T} A_{n}=$ I. If a polynomial $q \in \mathcal{P}$ is non-negative on $Z$, 
then,

$$
q=\sum_{j=1}^{k} f_{j}^{T} f_{j}+g,
$$

where $g \in I(Z)$ and $f_{1}, \ldots, f_{k} \in \mathcal{P}$.

Proof. We prove the contrapositive. Accordingly, suppose $q$ does not have the representation given in the theorem, that is $q$ does not belong to $\mathcal{C}_{2 c}(Z)$ for any $c \geq 1$. In pursuit of a stronger theorem, let $d-1$ denote the degree of $q$. Then $q \notin \mathcal{C}_{2 d}+I(Z)$ and so $\pi(q) \notin \mathcal{C}_{2 d}(Z)$.

By Minkowski's separation theorem there exists a linear functional $L_{1}$ on $\mathcal{P}_{2 d}$ satisfying:

$$
L_{1}(q)<0 \leq L_{1}(c), \quad c \in \mathcal{C}_{2 d}(Z) .
$$

Moreover, we can add to $L_{1}$ a small positive multiple of the functional $N$ appearing implicitely in the proof of Lemma 3.2:

$$
N(h)=\sum_{j=1}^{k}\left\langle h\left(B_{j}\right) \xi, \xi\right\rangle .
$$

To the effect that $L=L_{1}+\epsilon N$ satisfies $L\left(f^{T} f\right)>0, f \in \mathcal{P}_{d}(Z) \backslash\{0\}$.

Let $\Lambda$ denote the functional on $\mathcal{P}_{2 d}$ obtained by pulling back $L$, that is $\Lambda(r)=L(\pi(r))$. Define a symmetric positive semi-definite form on $\mathcal{P}_{d}$ by

$$
\langle a, b\rangle=\frac{1}{2} \Lambda\left(a^{T} b+b^{T} a\right)
$$

and let $H$ denote the associated Hilbert space . As a matter of fact, since the functional $\Lambda$ is strictly positive, $H=\mathcal{P}_{d}(Z)$ as a vector space. Use the notation $[p]$ to indicate the class of a polynomial $p \in \mathcal{P}_{d}$ in $H$ and let $\mathcal{M}$ denote the subspace of $H$ determined by $\mathcal{P}_{d-1}$. Thus, $\mathcal{M}$ is the set $\left\{[p]: p \in \mathcal{P}_{d-1}\right\}$. Let $\mathcal{N}$ denote the orthogonal complement of $\mathcal{M}$ in $H$.

Define $X_{j}$ and $Y_{j}$ as linear maps from $\mathcal{M}$ to $H$ by $X_{j}([p])=\left[x_{j} p\right]$ and $Y_{j}([p])=\left[x_{j}^{T} p\right]$. These mappings are well defined as $[p]=0$ if and only if $p \in I(Z)$ and $I(Z)$ is an ideal. Observe, for $a \in \mathcal{M}$,

$$
\begin{aligned}
\langle a, a\rangle-\sum\left\langle X_{j}^{T} X_{j} a, a\right\rangle & =\Lambda\left(a^{T} a\right)-\sum \Lambda\left(\left(x_{j} a\right)^{T}\left(x_{j} a\right)\right) \\
& =\Lambda\left(a^{T}\left(1-\sum x_{j}^{T} x_{j}\right) a\right)=0,
\end{aligned}
$$

where the last equality follows from the fact that $a^{T}\left(1-\sum x_{j}^{T} x_{j}\right) a$ is in $I(Z)$. Further, if $a, b \in \mathcal{M}$, then

$$
\begin{aligned}
<X_{j} a, b> & =<x_{j} a, b> \\
& =\frac{1}{2}\left(\Lambda\left(b^{T} x_{j} a\right)+\Lambda\left(\left(x_{j} a\right)^{T} b\right)\right. \\
& \left.=\frac{1}{2}\left(\Lambda\left(\left(x_{j}^{T} b\right)^{T} a\right)\right)+\Lambda\left(a^{T}\left(x_{j}^{T} b\right)^{T}\right)\right) \\
& =<a, Y_{j} b>.
\end{aligned}
$$


Thus, the restriction of $X_{j}^{T}$ to $\mathcal{M}$ is $Y_{j}$; i.e., $X_{j}^{T}$ on $\mathcal{M}$ is multiplication by $x_{j}^{T}$.

The computation in (6) shows that the mapping

$$
X: \mathcal{M} \rightarrow H \oplus H \oplus \ldots \oplus H
$$

defined by

$$
X[p]=\left(\begin{array}{c}
X_{1}[p] \\
\oplus \\
X_{2}[p] \\
\vdots \\
\oplus \\
X_{n}[p]
\end{array}\right)
$$

is an isometry. Since $\operatorname{dim} \mathcal{M}=\operatorname{dim}(X \mathcal{M})$, the map $X$ can be extended to an isometry $A$, defined on $H$ and with values in the direct sum $\oplus H$. Thus, $A=\left(A_{1}, \ldots, A_{n}\right)$ is an isometric tuple and the restriction of $A_{j}$ to $\mathcal{M}$ is $X_{j}$. Also, a simple computation shows that the operator $A_{j}^{T}$ compressed to $\mathcal{M}$ is equal to $Y_{j}$. More specifically, if $p, r \in \mathcal{M}$, then, using (7),

$$
\begin{aligned}
<A_{j}^{T} p, r> & =<p, A_{j} r> \\
& =<p, X_{j} r> \\
& =<X_{j}^{T} p, r> \\
& =<Y_{j} p, r>.
\end{aligned}
$$

Since the degree of $q$ is $d-1$, we have $q(A)[1]=q(X)[1]=[q]$. Also, $[q]=\left[q^{T}\right]$ because $q(B) \geq 0$ for every $B \in Z$. Therefore:

$$
\langle q(A)[1],[1]\rangle=\langle[q],[1]\rangle=\frac{1}{2}\left(\Lambda(q)+\Lambda\left(q^{T}\right)\right)=\Lambda(q)<0 .
$$

Thus, we have constructed an element $A \in Z$ such that $q(A)$ is not PSD.

The proof gives a marginally stronger result than stated. Namely, if $q$ has degree $d-1$ and is not in $\mathcal{C}_{2 d}(Z)$, then $q$ is not in $\mathcal{C}_{2 d^{\prime}}(Z)$ for any $d^{\prime} \geq d$. In other terms:

Corollary 4.2. For the class $Z$ of spherical isometries and any degree $d$ :

$$
\mathcal{C}_{2 d^{\prime}}(Z) \cap \mathcal{P}_{d-1}(Z)=\mathcal{C}_{2 d}(Z) \cap \mathcal{P}_{d-1}(Z), \quad d^{\prime} \geq d
$$

This observation gives a quantitative version of Theorem 4.1, in the following sense. Let $d-1$ be the degree of the polynomial $q$. Then $q$ does not belong to $\mathcal{C}(Z)$ if and only if $q$ does not belong to $\mathcal{C}_{2 d}(Z)$; and in this case the proof of Theorem 4.1 has produced a tuple $A$ in $Z$, acting on the Hilbert space $H=\mathcal{P}_{d}(Z)$ and with the property that $q(A)$ is not PSD. Thus, by negation, if $q(B) \geq 0$ for all isometric tuples acting on the Hilbert space $H$, 
then $q(C) \geq 0$ for every isometric tuple $C$, regardless of the dimension of the Hilbert space where $C$ acts. Now, it is easy to evaluate:

$$
\operatorname{dim} \mathcal{P}_{d}(Z) \leq \operatorname{dim} \mathcal{P}_{d} \leq(2 n)^{d} .
$$

In conclusion, we have proved the following result.

Proposition 4.3. Let $q$ be a non-commutative polynomial of degree $d$, in the variables $\left(x_{1}, \ldots, x_{n}, x_{1}^{T}, \ldots, x_{n}^{T}\right)$. Then $q$ admits the representation (4) if and only if $q(A) \geq 0$ for all isometric n-tuples $A$ of operators acting on a real Hilbert space of dimension $(2 n)^{d+1}$.

We have to point out that, in the commutative case, (i.e. polynomial algebra with trivial involution $x_{j}^{T}=x_{j}$ ) Theorem 4.1 is not true in dimension $n \geq 4$. Indeed, in this situation $Z$ would be the unit sphere in $\mathbf{R}^{n}$, and for instance Motzkin's counterexample can then be localized to any point of $Z$. See [12] for details.

4.2. Other supporting varieties. The proof of Theorem 4.1 can be adapted to the following situations:

a. The set $Z$ consists of all tuples of operators. In which case $I(Z)=0$ and Theorem 4.1 gives the main result of [4].

b. Let $S_{1}, S_{2}, \ldots, S_{k}$ be a partition of the set $\{1,2, \ldots, n\}$. The set $Z$ consists of tuples $\left(A_{1}, A_{2}, \ldots, A_{n}\right)$ of operators satisfying:

$$
\sum_{j \in S_{i}} A_{j}^{T} A_{j}=I, \quad 1 \leq i \leq k .
$$

In particular this covers the case $S_{i}=\{i\}, 1 \leq i \leq n$. That means that each $A_{j}$ is an isometry, and on a finite dimensional space we infer that each $A_{j}$ is an orthogonal transformation.

Similarly we can impose such isometric conditions only to a part of the $n$ tuple $A$, for instance to $\left(A_{1}, A_{2}, \ldots, A_{m}\right)$ with $m<n$.

c. Let $Q$ be a quadratic form in $n$ variables, with real coefficients. Let $Z$ be the set of $n$-tuples $A$ of real matrices subject to the condition:

$$
Q\left(A^{T}, A\right)=E
$$

where $E=0$ or $E=I$. Above we make the convention that all $A_{j}^{T}$ appear to the left of $A_{k}$.

Then Theorem 4.1 remains true, because a tuple $A$ of matrices satisfying condition (9) on a subspace $\mathcal{M}$ of a real Hilbert space $H$ can be extended to the whole $H$. The proof of the last assertion consists in diagonalizing (over the reals) the form $Q$, and extending the new tuple. More precisely, if $A$ admits the partition into sub-tuples $A=\left(A_{1}, A_{2}, A_{3}\right)$ and

$$
Q\left(A^{T}, A\right)=A_{1}^{T} A_{1}-A_{2}^{T} A_{2}
$$


then the relation:

$$
A_{1}^{T} A_{1}-A_{2}^{T} A_{2}=0,
$$

can be extended trivially by zero, while the relation

$$
A_{1}^{T} A_{1}-A_{2}^{T} A_{2}=I,
$$

can be extended as follows: $A_{1}$ is extended by an isometric tuple on $\mathcal{M}^{\perp}$ and $A_{2}$ is extended by zero.

\section{REFERENCES}

[1] F. Acquistapace, C. Andradas and F. Broglia, The strict Positivstellensatz for global analytic functions and the moment problem for semianalytic sets, Math. Ann. 316(2000), 609-616.

[2] J. Bochnack, M. Coste and J.-F. Roy, Géométrie Algébrique Réele, Springer, New York, 1987.

[3] G. Cassier, Probl eme des moments sur un compact de $\mathbf{R}^{n}$ et décomposition de polynomes a plusieurs variables, J. Funct. Analysis 58(1984), 254-266.

[4] J.W. Helton, Positive non commutative polynomials are sums of squares, Ann. Math. 56(2002), 675-694.

[5] J.W.Helton and S.A. McCullough, A Positivstellensatz for non-commutative polynomials, preprint 2002.

[6] M.G. Krein and A.A. Nudelman, The Markov Moment Problem and Extremal Problems, Transl. Math. Mono. Vol. 50, Amer. Math. Soc., Providence. R.I., 1977.

[7] S.Kuhlman and M. Marshall, Positivity, sum of squares and the multi-dimensional moment problem, Trans. Amer. Math. Soc. 354(2002), 4285-4301.

[8] S. McCullough, Factorization of operator-valued polynomials in several noncommuting variables, Linear Alg. Appl. 326(2001), 193-203.

[9] A. Prestel and C.N. Delzell, Positive Polynomials, Springer, Berlin, 2001.

[10] M. Putinar and F.-H. Vasilescu, Solving moment problems by dimensional extension, Ann. Math. 149(1999), 1087-1107.

[11] B. Reznick, Sums of even powers of real linear forms, Mem. Amer. Math. Soc. 96(1992), Amer. Math. Soc., Providence, R.I.

[12] C. Scheiderer, Sums of squares of regular functions on real algebraic varieties, Trans. Amer. Math. Soc. 352(2000), 1039-1069.

[13] K. Schmüdgen, On the moment problem of closed semi-algebraic sets, J. reine angew. Math., to appear.

[14] G. Stengle, A Nullstellensatz and a Positivstellensatz in semialgebraic geometry, Math. Ann. 289(1991), 203-206.

Department of Mathematics, University of California at San Diego, La Jolla CA 92093

E-mail address: helton@osiris.ucsd.edu

Department of Mathematics, University of Florida, Gainesville, FL 326118105

E-mail address: sam@mail.math.ufl.edu

Department of Mathematics, University of California, Santa Barbara, CA 93106

E-mail address: mputinar@math.ucsb.edu 